Journal of Mathematics and Statistics 4 (1): 58-59, 2008

ISSN 1549-3644

(C) 2008 Science Publications

\title{
A New Approach for Solving Second Order Ordinary Differential Equations
}

\author{
Laith K. AL-Hwawcha and Namh A. Abid \\ Al-Zaytoonah University, Amman, Jordan
}

\begin{abstract}
A new approach is presented to solve second order linear differential equations with variable coefficients and some illustrative examples are given.
\end{abstract}

Key words: Second order equations, general solution, homogeneous and nonhomogeneous equations

\section{INTRODUCTION}

Consider the second order linear ordinary differential equation

$$
y^{\prime \prime}+P(x) y^{\prime}+Q(x) y=G(x)
$$

where, $\mathrm{P}, \mathrm{Q}$ and $\mathrm{G}$ are continuous functions. It is known that the power series method is a powerful method for solving Eq.(1). However, this method needs a lot of time, space and high concentration during calculations. In this research, we present a new approach which can be used to a wide class of equations either to find a general solution to the associated homogeneous equation or to find a particular solution to Eq.(1) without requiring the general solution or any solution of the associated homogeneous equation as most methods require. For more details, see[1].

\section{MAIN RESULTS}

In this section we introduce our main results.

Theorem 1: Consider the equation

$$
y^{\prime \prime}+P(x) y^{\prime}+Q(x) y=0
$$

If $v(x)=y^{\prime}(x)+\beta(x) y(x)$, where $\beta(x)$ is a solution of the Riccati equation $\beta^{\prime}(x)=Q(x)-P(x) \beta(x)+\beta^{2}(x)$, then,

$$
y(x)=e^{-\int \beta(x) d x} \int e^{\int(2 \beta(x)-P(x)) d x} d x
$$

is a solution of Eq.(2).

Proof: It is easy to show that $\mathrm{v}^{\prime}=(\beta(\mathrm{x})-\mathrm{P}(\mathrm{x})) \mathrm{v}$, where Riccati equation has been used and $v(x)=e^{\left.\int \beta(x)-P(x)\right) d x}$, then the result is achieved.
Note: It is known that the substitution $v(x)=\frac{-y^{\prime}}{y}$ transfers Eq. (2) to a Riccati equation and $y=e^{-\int v(x) d x}$ is a solution of the equation. This result is included in the theorem (1) and the formula (3) really gives a second linearly independent solution to Eq. (2) and therefore the general solution is constructed. These facts are illustrated in the following example.

Example 1: Find a general solution of the equation

$$
x y^{\prime \prime}-(1+x) y^{\prime}+y=0
$$

Solution: Here, $\mathrm{P}(\mathrm{x})=\frac{-(1+\mathrm{x})}{\mathrm{x}}, \mathrm{Q}(\mathrm{x})=\frac{1}{\mathrm{x}}$, so the Riccati equation is

$\beta^{\prime}(\mathrm{x})=\frac{1}{\mathrm{x}}+\left(\frac{1+\mathrm{x}}{\mathrm{x}}\right) \beta(\mathrm{x})+\beta^{2}(\mathrm{x})$

and $\beta(x)=-1$ is a solution of the equation, and then $y_{1}(x)=e^{\int d x}=e^{x}$ is a solution of the equation. Thus

$$
\begin{aligned}
y_{2}(x) & =e^{\int d x} \int e^{\int\left(-2+\frac{1+x}{x}\right) d x} d x \\
& =-x-1 .
\end{aligned}
$$

Hence the general solution is

$$
\mathrm{y}(\mathrm{x})=\mathrm{c}_{1} \mathrm{e}^{\mathrm{x}}+\mathrm{c}_{2}(\mathrm{x}+1)
$$

By using the same technique, naturally one can get the following result, which can be used to find a particular solution of Eq. (1). In particular, this procedure can be used easily to find a particular solution of second order ordinary differential equations 
with constants coefficients and for Cauchy- Euler equation because the associated Riccati equation is solvable.

Theorem 2: Consider the equation

$$
y^{\prime \prime}+P(x) y^{\prime}+Q(x) y=G(x)
$$

If $\mathrm{v}(\mathrm{x})=\mathrm{y}^{\prime}(\mathrm{x})+\beta(\mathrm{x}) \mathrm{y}(\mathrm{x})$, where $\beta(\mathrm{x})$ is a solution of the Riccati equation

$$
\beta^{\prime}(\mathrm{x})=\mathrm{Q}(\mathrm{x})-\mathrm{P}(\mathrm{x}) \beta(\mathrm{x})+\beta^{2}(\mathrm{x}),
$$

then

$$
y(x)=e^{-\int \beta(x) d x} \int\left(e^{\int(2 \beta(x)-P(x)) d x} \int G(x) e^{-\int(\beta(x)-P(x)) d x} d x\right) d x
$$

is a solution of Eq. (5).

Example 2: Find a particular solution of the equation

$$
x^{2} y^{\prime \prime}+3 x y^{\prime}+y=x^{2} \ln x, x>0
$$

Solution: Here, $\mathrm{P}(\mathrm{x})=\frac{3}{\mathrm{x}}$ and $\mathrm{Q}(\mathrm{x})=\frac{1}{\mathrm{x}^{2}}$, so the Riccati equation is given by:

$$
\beta^{\prime}(x)=\frac{1}{x^{2}}-\frac{3}{x} \beta(x)+\beta^{2}(x),
$$

and $\beta(x)=\frac{1}{x}$ is a solution of the equation. Thus

$$
\begin{aligned}
y_{p}(x) & =e^{-\int \frac{1}{x} d x} \int\left(e^{-\int \frac{1}{x} d x} \int \ln (x) e^{2 \int \frac{1}{x} d x} d x\right) d x \\
& =\frac{1}{9} x^{2}\left(\ln (x)-\frac{2}{3}\right)
\end{aligned}
$$

is a particular solution of the given equation.

\section{CONCLUSION}

In this research we introduce a new approach for solving second order ordinary differential equations, and it seems an easier way to teach these equations than the usual ones.

\section{REFERENCES}

1. Boyce, W.E. and R.C. DiPrima, 2000. Elementary Differential Equations and Boundary Value Problems. John Wiley and Sons, Inc. 